\title{
LA DISCRIMINACIÓN EN EL APRENDIZAJE DEL CUIDADO ${ }^{1}$
}

\section{DISCRIMINATION IN LEARNING NURSING CARE}

\author{
Ricardo Ayala Valenzuela* \\ Catalina Alvarado-Negrón ${ }^{* *}$ \\ José CUEVAS-AzÓCAR ${ }^{* * *}$
}

\begin{abstract}
RESUMEN
Esta investigación tuvo por objeto comprender el fenómeno de la discriminación como parte del aprendizaje del cuidado en estudiantes de enfermería, vale decir, comprender de qué manera experimentan el fenómeno de escoger o priorizar a un individuo sobre otro al momento de dar cuidados. Investigación cualitativa, en la que los datos fueron recopilados mediante entrevistas semiestructuradas complementadas mediante el registro de notas de campo. Muestra intencionada compuesta por estudiantes de enfermería de una universidad del sur de Chile, quienes se encontraban cursando el tercer y cuarto año del plan de estudios, y que habían aprobado los cursos profesionales. Hecho esto, aplicamos el método de casos extremos en base al rendimiento académico como criterio principal. Con apoyo de la herramienta Atlas.ti, los datos acopiados fueron sistematizados y reducidos empleando como guía la Teoría Fundamentada en los datos, llegando a emerger cuatro categorías analíticas. Se concluye que los estudiantes realizan una discriminación en favor de sí mismos, fundamentalmente en base a la patología del sujeto de atención y a factores personales de éste, tales como rostro amable, apertura al diálogo, respuesta comunicativa, entre otros. Encontramos en ellos especial interés por las personas con patologías que les resultan interesantes, conocidas, o bien, aquéllas en las que se presagia una estadía suficientemente larga como para participar de la evolución y evaluar los resultados de un plan de cuidados. Estos elementos surgieron como determinantes en las emociones que producen en los aprendices, instándoles a dedicar un mejor cuidado a unas personas que a otras.
\end{abstract}

Palabras clave: Enfermería, cuidado, aprendizaje, discriminación, toma de decisiones, estudiantes de enfermería.

\begin{abstract}
The purpose of the research was to understand the phenomenon of "discrimination" as a part of nursing students learning how to provide nursing care, that is, how students make decisions and prioritize nursing care. Using a qualitative research methodology data collection included the use of semi-structured interviews and field notes. The sample was made up of nursing students enrolled in a nursing program in a university in southern Chile. The students had acquired specific clinical skills and were in the third and fourth years of study. The extremecase method was used based on students' academic scores as mean criterion. Data were analyzed using the Atlas. ti tool followed by application of Grounded Theory techniques to identify four analytical categories. Results showed that nursing students were able to discriminate for themselves, based mainly on criteria about disease and personal factors, such as the attractiveness of a person's face, openness to dialog, level of communication and so on. Findings showed that students paid special attention to people with 'interesting' diseases, known diseases, or health problems which offered students the opportunity to implement nursing care plans and to assess outcomes. These elements emerged like determinant factors based on the emotions that they produce in the beginning students, triggering them to give better care to some individuals over others.
\end{abstract}

Key words: Nursing, caring, learning, discrimination, decision making, nursing students.

Fecha recepción: 17/03/09 Fecha aceptación: 10/08/2010

\footnotetext{
${ }^{1}$ Este artículo es una ampliación y profundización de una línea argumental de una investigación mayor, correspondiente a la Tesis de Grado de dos de los autores, la cual fue realizada en 2008 en la Universidad Austral de Chile.

* Enfermero. E-mail: RicardoAlexis.AyalaValenzuela@Ugent.be

** Enfermera. Hospital de la Mutual de Seguridad. Santiago de Chile. E-mail: catalina.alvarado@alumnos.uach.cl

${ }^{* * *}$ Enfermero. Hospital Base de Osorno. Osorno, Chile. E-mail: jose.cuevas@alumnos.uach.cl
} 


\section{INTRODUCCIÓN}

Para poder aproximarnos al tema del estudio, nuestra revisión teórica se ha basado en tres ejes principales, en los que cimentamos nuestros puntos referenciales. En primer lugar, hacemos una revisión a la corriente del cuidar-cuidado (caring), para luego dar paso a los mecanismos de aprendizaje de seres humanos aplicados al acto de cuidar. Por último, abordamos el proceso de discriminación como un tipo de aprendizaje, en el marco de la toma de decisiones que los estudiantes de enfermería realizan en sus experiencias de aprendizaje, mostrando evidencias que hacen de antecedente al problema de estudio.

\section{El cuidado}

Es ampliamente aceptado que el cuidado contiene la reverencia por la vida y el amor de sí mismo, el ver a otros como seres que tienen un poder para crecer y cambiar de forma trascendental, capaces de convivir plásticamente con el tiempo, el espacio y la corporeidad; y por otro lado, que la enfermería es proporcionar ayuda no paternalista para el desarrollo de éstos y otros aspectos para preservación de la humanidad, cuestión que constituye el marco espistémico del cuidar de otros $(1,2)$.

La importancia que el cuidar adquiere para esta disciplina ha sido reconocida desde los orígenes de su práctica. Nightingale reconoce que la enfermería crea la atmósfera curativa y del cuidado, lo que contribuye positivamente a la recuperación de las personas a través de la curación. En tanto, Watson lo define como "el ideal moral de la enfermería y cuyo fin es la protección, elevación y preservación de la dignidad humana" (3). Sus postulados revelan gran influencia de las teorías de Nightingale, Henderson, Krueter, Hall y Leininger, así como las de Carl Rogers, Erickson y Maslow (4). La misma autora plantea que el cuidado emerge desde un sis- tema de valores humanistas y altruistas que ayuda a tolerar las diferencias y a ver al otro a través de su sistema perceptual, más que a través del propio.

Watson, al hablar de salud, se refiere a la unidad y armonía entre la mente, el cuerpo y el alma, relacionándose con el grado de coherencia entre lo que percibe y experimenta uno mismo y su yo. Para ello propone diez factores centrados en las cualidades de quien otorga cuidados y las fuerzas fenomenológicas que es capaz de movilizar en otros. Lejos de una óptica fragmentada (la del ser 'biopsico-social'), el centro de la enfermería está en el conocimiento de la persona como ser completo e infragmentable, un ser humano unitario (5).

Los postulados de Watson se complementan con el planteamiento de Carper (6), quien sostiene que el conocimiento de enfermería no reside únicamente en las evidencias científicas, en los datos empíricos obtenidos mediante el método científico ni a través de la racionalidad impuesta por la aplicación de tal método en el cuidado mediante el proceso de cuidar. Afirma que la práctica tiene también otras fuentes de conocimiento, tales como la vida emocional e intuitiva dada por el desarrollo de sí mismo como instrumento terapéutico (conocimiento personal), de la experiencia de probar límites del bien y el mal (conocimiento ético), y del adecuar permanentemente el arte de cuidar a las necesidades instrumentales y trascendentales de otros (conocimiento estético), en un proceso creativo que no puede llegar a mecanizarse. Diversos autores reconocen los patrones de conocimiento propuestos por Carper, como un marco conceptual para la práctica de enfermería, la educación y la investigación (7-13).

Entonces, en la era profesional del desarrollo de la disciplina de enfermería ésta se aleja cada vez más de una visión 'hospitalcéntrica' descrita por Watson (14). Así también, la educación en enfermería se ha modificado, migrando desde los muros del hospital a las aulas de la universidad, lo que da lugar a una 
disciplina centrada en adquirir conocimientos para comprender la conducta humana. Por tanto, las actitudes del cuidado deben ser enseñadas en los primeros años de estudios, y ser reforzadas en los años que siguen, despertando en el estudiantado un genuino 'deseo de cuidar'.

En su Teoría del Cuidado Humano, Watson presenta también una visión del cuidado como factor integrador en el desarrollo del curriculum de enfermería (3).

\section{Aprendizaje del cuidado}

El aprendizaje, por su parte, ha estado explicado fundamentalmente por las teorías psicológicas. Por un lado, desde una perspectiva conductista (fuertemente arraigada a nuestra tradición educacional), éste se hace evidente con la realización de una conducta, un movimiento, un acto, y se consolida mediante un estímulo que refuerza negativa o positivamente. Esta óptica - muy limitada, por cierto-es complementada con posterioridad mediante la comprensión de los procesos de pensamiento mediados por el desarrollo de la capacidad de abstracción en etapas secuenciales. Por su parte, el enfoque humanista explica el aprendizaje como resultado de una necesidad que opera en el mecanismo de la motivación, y el constructivista como el resultado de una experiencia social (15).

Pese a las grandes dificultades que implica aventurar una definición certera y acabada del concepto de aprendizaje (16), nos apoyamos en la existencia de un proceso interno de adquisición del conocimiento y, por tanto, no observable (17). Se desprende de esto que es posible llegar a él sólo de forma indirecta, a través de una selección de muestras que proponemos como evidencia de determinado aprendizaje en un diseño curricular.

Ya lo señala Ardilla (18): "en español, y en los demás idiomas, el término 'aprendizaje' es bastante equívoco e implica dos significados diferentes, aunque íntimamente relacionados: la adquisición de información o de habilidades motoras, por ejemplo aprender matemáticas o aprender a conducir un auto; y aprendizaje como modificación del comportamiento por la experiencia previa[...]". En contraposición, el mismo autor discute abordar el aprendizaje desde la pura conducta o el movimiento; por ejemplo, las plantas tienen un movimiento estimulado por la luz del sol, por el toque táctil u otros estímulos cuyo aparato sensorial les permite percibir. Esto explica la apertura y cierre de los pétalos florales y de otros 'comportamientos', como el citado por él mismo sobre aquellas especies pertenecientes al género Mimosa. Pareciera ser, entonces, que el comportamiento no explica el aprendizaje por sí solo, pues en él se involucran otras funciones realmente cerebrales, tales como el aprendizaje del animal que logra diferenciar entre uno y otro objeto, entre una y otra persona; en definitiva, un aprender a discriminar.

Arribamos, entonces, a una comprensión del concepto aprendizaje como un constructo hipotético relativo a un cambio interno, que algunas veces se manifiesta de forma indirecta en un comportamiento. Así, también puede ser entendido como un "proceso de adquisición cognoscitiva que explica, en parte, el enriquecimiento y la transformación de estructuras internas, de las potencialidades del individuo para comprender y actuar sobre su entorno, de los niveles de desarrollo que contienen grados específicos de potencialidad" (19).

Lo relacionado con la conducta y el cambio de ésta, según ya hemos insinuado, es limitado, en tanto involucra también procesos más complejos, como lo llama Biggs "una forma de interactuar con el mundo", pues en el curso de aprender "cambian nuestras concepciones de los fenómenos y vemos el mundo de manera diferente" (20). Cuando aprendemos se activan mecanismos y procesos cognitivos que nos permiten construir representaciones sobre el mundo, pero también imaginar o proyectar nuevos mundos posibles, dando a lo que nos rodea "nuevos significados". Como 
lo plantea Pozo "sería por tanto el mundo el que constituiría un reflejo del conocimiento construido, y no al revés" (21), bien desde un enfoque analítico por descomposición del entorno en partículas simples, bien por la significación simbólica con que, en su conjunto, esos elementos ganan un significado dentro del proceso de interpretación. Tanto una como otra forma de aprender están relacionadas con la autopercepción de progreso, la voluntad de poder y, como un aprendizaje en sí mismo, el querer aprender.

En los procesos cognitivos que median en el aprendizaje operan algunos elementos, tales como a. la imagen, reproducción mental relevante en el proceso de recuerdo y evocación; b. el raciocinio, interrelación semántica entre juicios; c. el pensamiento, procesos cognitivos caracterizados por el uso de símbolos; d. la inteligencia, una estimación del funcionamiento intelectual; e. el lenguaje, facultad o capacidad del ser humano de comunicarse y de representar la realidad mediante signos; y f. un componente afectivo, determinante de las condiciones de sujeto para poder aprender, tales como la empatía, las emociones y los sentimientos (17).

Una corriente muy actual en educación y otras ciencias tanto humanistas como biológicas es la emocionalidad. "La educación emocional y social implica el aprendizaje de habilidades, conocimientos y valores que aumentan nuestra capacidad de 'leer' en nosotros mismos y en los demás con el fin de usar esa información para resolver problemas con flexibilidad y creatividad" (22). Las emociones influyen en la forma e intensidad de aprender, particularmente dentro de la relacionalidad que se forja con otras personas, en la atmósfera educacional y en el desarrollo de oportunidades de aprendizaje (23-28), que generan mucho más que aprendizajes: aprendizajes verdaderamente significativos, útiles en resolver complejidades, transformar el entorno y regular la propia emotividad.

Como podemos deducir, son múltiples los caminos que podemos recorrer junto al sujeto de aprendizaje, facilitando u obstaculizando sus procesos mentales. Como lo señala Maya, obstáculos epistemológicos, conceptuales, actitudinales u operativos que pueden bloquear una educación ciertamente integral (29).

Por su parte, Valenzuela plantea que el aprendizaje que deben realizar los estudiantes, sean escolares o universitarios, ha de ser profundo, cuestión que implica la construcción y establecimiento de una red de conexiones significativas, tanto dentro de una disciplina como en su relación con otros dominios de conocimientos y, por supuesto, también con la vida cotidiana (30).

Entonces, ¿cómo es que los estudiantes llegan a aprender el cuidado? El que los estudios de enfermería estén en la actualidad vinculados a la universidad, directa o indirectamente, implica su involucramiento con el desarrollo teórico, científico y tecnológico. Sin embargo, al tratarse de una disciplina de carácter práctico, cobra importancia el aprendizaje experiencial, lo que resulta de la participación directa en situaciones del ejercicio, en una perspectiva que acompasa la percepción, la cognición y la conducta. Entonces, quien aprende puede directamente participar de la experiencia y, subsecuentemente, puede adquirir algún tipo de nuevas competencias identificables (31).

La evidencia actual pareciera apuntar hacia un fenómeno de adaptación del estudiante a situaciones nuevas, pero no así al mecanismo de cómo es que llega a ser aprendido o qué significado adquieren tales experiencias. De esto se desprende la dificultad en la que se encontrará cuando deba enfrentar un dilema en que deba optar por un curso de acción, pues casi todas las decisiones que enfermería toma corresponden a una dimensión moral (32). Un factor agravante se nuclea en que la reflexión, como acto pedagógico, aún no se encuentra masificado en el ámbito de la enfermería (33), cuestión que es necesaria corregir, tanto en la práctica como en la educación en enfermería, mediante una relación 
pedagógica reflexiva, proyectando conocimientos nuevos o imaginando una práctica deseable.

Los mismos autores plantean que cada acto del 'buen cuidar' está afectado de una u otra forma por las propias emociones. Una persona es tocada por una situación y actúa en consecuencia, tratando de evitar lo que le resulta desagradable. Esto nos lleva a una de las cuestiones medulares del aprendizaje emocional, en tanto la práctica del cuidado requiere de las enfermeras un aparato sensitivo extremadamente sensible a las necesidades de cuidado. Por tanto, el estudiante que aprende a cuidar se mueve en una constante entre la sensibilidad extrema y la indiferencia. Según las observaciones de Greenwood (34) existe en el aprendizaje de cuidar un importante componente no intencional, ligado a las emociones vividas, pero que es obscurecido por la racionalización que bloquea una respuesta emotiva espontánea, esto es para nosotros un plano fenomenológico constreñido por el plano lógico.

En otro ámbito, se reconoce un importante segmento del aprendizaje enfermero como una participación social, en el que fenómenos son descubiertos en la práctica de forma azarosa y muchas veces deslumbrante $^{2}(35)$, lo que en educación suele denominarse insight: el llegar a descubrir de manera inesperada y sorpresiva algo que siempre se quiso comprender. Quizás este evento podría estar relacionado con lo que se plantea como un quinto patrón de conocimiento en enfermería: el desconocimiento, entendido como la apertura a nuevas ideas, saberes y creencias (36). Los marcos de experiencia, entonces, construyen parte del saber de enfermería pero también de su identidad, una aculturación que permite llegar a ser aceptados en el equipo de trabajo, fundiéndose y confundiéndose en su sistema de creencias y valores. Estas experiencias clínicas confor- man un factor gravitante en los programas universitarios de enfermería, ampliamente estudiado $(35,37,38)$.

En la propia relación con el sujeto de cuidados, inmerso en la práctica, las emociones funcionan como un engrane fundamental. En la medida que un sujeto totalmente desconocido se va haciendo familiar aumenta el compromiso con éste, se visualiza como una persona desprotegida o en peligro, que requiere de una respuesta. "La participación que puede darse entre el profesional de enfermería y el paciente tiene un fuerte elemento emocional. Un paciente puede suscitar la compasión, la preocupación, la pena o incluso la ira o la frustración" (39). Para la propia autora, estas emociones juegan un papel en la motivación moral, en el juicio moral y en la acción de las enfermeras, inmiscuyéndose en la percepción que tenemos sobre las vicisitudes y pesares de otros, sobre los problemas que sobrevienen en seres humanos usualmente vulnerables. En esta misma línea, el trabajo de enfermería ha sido descrito como un 'trabajo emocional' (40), elemento que no se encuentra presente en las reglas de las organizaciones de salud ni en la descripción formal del trabajo, pero que forma parte esencial de la identidad de las enfermeras.

\section{Toma de decisiones al cuidar}

El tomar decisiones es inherente al desempeño de una profesión, cualidad que se adquiere principalmente durante las experiencias clínicas en contacto con los sujetos de cuidado, en el entorno laboral. Después de un tiempo dentro del bosque podemos diferenciar entre mil distintos tonos de verde. Así, agrupamos elementos con características similares dentro de 'casilleros mentales' o categorías, hecho que nos permite clasificar y comprender el entorno. "El aprendizaje y utilización de categorías constituye una forma de conocimiento más

\footnotetext{
${ }^{2}$ Los autores referenciados emplean el adverbio 'serendipitously', que no tiene una traducción literal a nuestra lengua.
} 
elemental y general por las que el hombre ${ }^{3}$ se adapta a su entorno" (41).

En 1970 Johansen definió la toma de decisiones como el acto de escoger entre diferentes alternativas a aquella que implique la acción más apropiada para la obtención de determinado objetivo (42). Tomar la mejor decisión supone haber hecho un análisis de lo que hubiera sucedido si cada una de las posibles alternativas se hubiera seleccionado. La toma de decisiones es un acto cotidiano que está involucrado en múltiples actividades que generalmente se hace por técnicas como la adivinanza, la reacción visceral, la intuición, o la experiencia basada en opiniones o sucesos muy parecidos. Pocas decisiones se toman con plena certidumbre sobre sus posibles consecuencias (43).

Los profesionales de salud enfrentan un sinnúmero de decisiones en el curso de su práctica (44). La etapa de estudios de grado procura desarrollar la habilidad de toma de decisiones relacionadas con la conducta y el manejo terapéutico de los pacientes; sin embargo, ésta presenta importantes limitaciones a causa de que el estudiante no puede tomar por sí solo muchas de estas decisiones por las posibles desfavorables consecuencias que pudieran acarrear para el paciente (45).

En la década de los 90 Valenzuela realiza una observación sin precedentes en Chile: la actitud del cuidado en estudiantes de enfermería varía en relación a nivel educacional que tiene el sujeto, experimentándose cierta tendencia hacia personas con nivel de educación bajo y mediano, hacia los adultos jóvenes y los maduros, hacia los que se comunican mejor, y a los que presentan cierto grado de dependencia ${ }^{4}$.

Fenómenos similares habían sido observados en los 70 por varios autores en el extranjero, quienes hallaron que las personas que estaban menos enfermas eran más deseadas para ser cuidadas por las enfermeras (46). Por el mismo tiempo también fue documentado que los individuos que tenían facilidad para comunicarse, por su personalidad o actitudes individuales, influían en que las enfermeras se sintieran agradadas de proporcionarles cuidado (47). También se encuentran otros estudios en los que el atractivo físico del sujeto se asocia con el cuidado que recibe (48, 49); los pacientes físicamente 'atractivos' fueron tipificados por los que los cuidaron como personas con mejor pronóstico y siendo 'más inteligentes' y 'motivados' que los físicamente 'menos atractivos'. Más tarde, hacia fines de los 80 se describió que los estudiantes de enfermería sentían agrado de entregar un mejor cuidado a los pacientes alegres y comunicativos, a quienes tenían dolor o percibían con una mayor necesidad de cuidado, como igualmente a los que respondieron positivamente al cuidado entregado (50).

\section{Problema de investigación}

La evidencia sugiere que los estudiantes de enfermería realizan actos racionales (emocionales), deliberados y conscientes que responden a su facultad de discriminar entre un sujeto y otro, como parte de un proceso de toma de decisiones por preferir o rechazar cuidarlos. Del análisis de estos elementos surge la importancia de comprender el modo en que este proceso de discriminación involucra a los sujetos de atención. Éste aflora como un hecho cardinal, en el sentido de que conociendo, reforzando y monitoreando esta etapa se puede actuar sobre ella, modificando los elementos que dificultan el aprendizaje y potenciando aquellos que lo facilitan.

Entonces, el problema de estudio se engloba en las preguntas: ¿De qué modos opera

\footnotetext{
${ }^{3}$ Hemos respetado la palabra 'hombre' dentro de la cita textual, aunque nosotros preferimos referirnos a la persona como 'ser humano'.

${ }^{4}$ Valenzuela S. Factores que influyen en la actitud de estudiantes de Enfermería hacia el cuidado de pacientes durante sus experiencias clínicas médico-quirúrgicas [Tesis Magíster]. Universidad de Concepción, Concepción, Chile; 1995.
} 
el fenómeno de la discriminación, como parte del proceso de toma de decisiones en estudiantes de enfermería, al momento de brindar cuidados a un sujeto de atención?, ¿cómo se da este proceso en el estudiante?, ¿qué factores se asocian al que un estudiante decida seleccionar a una u otra persona para dar cuidados?

\section{Objetivos}

Comprender las formas en que opera el fenómeno de la discriminación, como parte del proceso de toma de decisiones en estudiantes de enfermería, al momento de brindar cuidados a un sujeto de atención. Explorar el proceso racional de discriminación ejercido por los estudiantes de enfermería, al momento de realizar o brindar cuidados a un sujeto. Categorizar factores que se presentan como asociados al proceso de toma de decisiones en los estudiantes de enfermería al momento de brindar cuidados en el contexto nosocomial.

\section{DISEÑO METODOLÓGICO}

Corresponde a una investigación cualitativa (51), para la que se empleó la técnica de entrevista semiestructurada (52). Las entrevistas fueron realizadas a una muestra intencionada de estudiantes de enfermería de una universidad chilena, identificados como informantes representativos polares (53), que a su vez compartían características de informantes clave (52). Los criterios de conformación de la muestra intencionada estuvieron dados por promedio de calificaciones, así como el grado de avance en el plan de estudios. De este modo, incluimos estudiantes con altos y bajos promedios (aun cuando la diferencia no era amplia), cursantes de tercer y cuarto año de enfermería, con experiencias concretas sobre la toma de decisiones en el proceso de cuidar en variados contextos.

Se comenzó con una muestra constituida por dos informantes, aumentando gradualmente hasta lograr el punto de saturación de los datos (54), que fue advertido cuando la realización de nuevas entrevistas no arrojó variantes que proporcionaran nuevos elementos de interés para la comprensión del fenómeno estudiado (52). Esto sucedió al cabo de la décima entrevista, incluyendo hasta entonces un total de seis informantes cuyas calificaciones figuraban como las más altas, y cuatro entre las más bajas.

Los encuentros tuvieron lugar en un Hospital Clínico, con el propósito de aportar al entrevistado el contexto físico donde transcurrieron sus experiencias de aprendizaje.

Una vez obtenidos los discursos mediante dispositivo digital, fueron transliterados para obtener marcos hermenéuticos que fueron analizados empleando como guía a la Teoría Fundamentada (55), y sistematizados mediante Atlas/ti 5.0. Una vez codificados los datos, identificamos las categorías emergentes de análisis en las cuales organizamos los resultados para presentarlos.

\section{Consideraciones éticas}

Todos los participantes fueron entrevistados previo consentimiento informado. Sus nombres reales y los de personas aludidas fueron reservados y modificados, así como suprimidas situaciones que expusieran a terceros y el nombre de la universidad escogida como locación.

\section{RESULTADOS}

Los marcos hermenéuticos ${ }^{5}$ fueron codificados y organizados en cuatro categorías que emergieron de su análisis.

\footnotetext{
${ }^{5}$ Los registros completos de las entrevistas se mantienen en poder de los autores, tomando de ellos sólo las citas necesarias para ilustrar los resultados pertinentes a los objetivos de esta investigación. Los corpus de las entrevistas fueron codificados con una letra $C$ seguida del número correlativo, de acuerdo al orden de incorporación de los informantes en la muestra.
} 


\section{Proceso de aprendizaje vinculado al conocimiento sobre patología}

Los estudios universitarios implican ofrecer ambientes para aprender, practicar, conocer, equivocarse, y también para reflexionar sobre esa práctica, ese conocimiento y esas equivocaciones. En el discurso de los informantes aparecen elementos vinculados a lo experiencial de su aprendizaje, particularmente en lo relacionado al descubrimiento de los procesos de enfermedad y a la confirmación de la teoría en la práctica.

Nos parece evidente una selección de los sujetos en base a la complejidad de sus cuadros, lo que podría estar explicado por la percepción de mayores posibilidades de aprender mientras más alta sea la severidad del problema de salud. Pareciera ser que personas cuyos cuadros sean 'raros' podrían tener menos posibilidades de ser elegidos por un estudiante de enfermería para ser cuidado, que otro con cuadros que le sean más familiares o de una complejidad que le resulte 'emocionante'.

De uno u otro modo, aparece el elemento patología como determinante en la selección de un sujeto, centrado -seguramente- en las posibilidades que esas circunstancias ofrecerán, propiciando su alerta por aprender algo que resulte llamativo, o bien atenuando el efecto incómodo de situarse con pocos elementos frente a una persona enferma y a un entorno desconocido. Este elemento apareció con fuerza, tanto en estudiantes con altas como con bajas calificaciones.

C8: "Siempre me voy por el más complicado." / C2: "La patología que tal vez sea más compleja, donde pueda aprender más..." / C7: "Yo escogería al que tuviera patologías que yo maneje, que yo sepa de qué les voy a hablar, qué les voy a preguntar, cosas que ya he desarrollado..." / C5: “...que sea una patología quizás que a mí guste, que yo maneje... o que yo por lo menos haya escuchado, porque si es algo muy raro no me va a tincar ese paciente, ya con el nombre me va a complicar.” / C9: “...iría a atender a un paciente que tenga algo que yo sé, una patología que yo conozca...” / C4: “... que sea una patología en la que yo pueda aprender, que sea compleja..." / C10: “...sinceramente al que no esté tan complejo, y al que se vea mejor..." / C3: “...tenía una patología media extraña (sonrie), entonces dije, mmm interesante, y por eso la escogí, era un nombre medio extraño..." / C6: "...que sea que yo maneje y mucho si el paciente está bien, esta complicado, porque ahí tengo más cosas, en el fondo, que hacer..."

Probablemente, el padecer una patología de mayor complejidad y requerir una hospitalización más prolongada pueda ser visto como una oportunidad para crear planes de cuidados a mayor plazo, visualizándose como una posibilidad de realizar un seguimiento del paciente y poder confirmar o rechazar los resultados de las propias acciones y de otros, y de poder sentir satisfacción de contemplar el trabajo hecho.

C4: “...me guío por patologías que estén más tiempo en el hospital.” / C7: “...un día tenía todo listo... al otro día volvía con mi planificación hecha, y lo habian dado de alta. Entonces, es como ese el sentimiento que tengo, el conocerlo bien, tener la sensación que ese paciente se va de alta y no haberle hecho la atención completa, como que eso no me deja satisfecha..."

\section{Comunicación terapéutica y relación de ayuda}

Quizás uno de los factores más gravitantes que parecen favorecer el bienestar emocional del estudiante está dado por el proceso de comunicación que logre con el sujeto de atención, posiblemente ligado a un mecanismo de probarse como instrumento terapéutico, o bien como una forma de comprender de mejor manera lo relacionado con el proceso patológico que cursa la persona, sus factores desencadenantes y el curso de la enfermedad, como también lograr aproximarse a las 
respuestas que su condición humana genera ante los problemas de salud.

C5: “...me gusta hablarles, entonces como que trato de investigar de su vida, vamos conversando y solitas van saliendo cosas..." / C7: “...escogí a la señora que escogí porque, bueno, me di cuenta que ella hablabay era más fácil para mí establecer un dialogo con ella..." / C8: "Yo creo que de repente lo que me favorece un poco más, es que yo les hablo en su idioma."

De acuerdo a lo anterior, es posible observar que los estudiantes experimentan una preferencia por los sujetos de atención que tienen mayor facilidad para comunicarse de forma oral y por quienes tienen una historia de vida que consideran interesante y llamativa al momento de la valoración, en términos de lo que puede ser beneficioso en su proceso de aprender. Sin embargo, el que condiciones como lucidez, condición mental o habilidad de comunicación puedan ser también afectados por la severidad del cuadro podría hacer enfrentar una disyuntiva interesante de explorar.

C8: "cuando uno ve a alguien le parece más tiernito que otro, ¿Te fijas?, por eso sería, o de repente conversar con cada uno y la historia que me parezca más interesante, la historia quizás de vida o apreciación psicológica que me parezca un poco más interesante...” / C8: “... hay personas que tienen la tremenda historia de vida y que uno siente como una conexión de buena onda con esas personas, entonces uno igual trata de trabajar con ellos...” / C1: “... soy una persona que conversa harto, pero también pregunto harto (sonrie), que me cuenten de cómo han andado, cómo han dormido, cómo han estado, todas esas cosas que uno hace de su valoración antes de una atención." / C5: "Prefiero una orientada, que una desorientada." / C5: “...no me gustan tanto por ejemplo los típicos borrachitos, que empiezan con sus convulsiones y no entienden nada de lo que uno les habla [...] Y en el fondo no puedes trabajar mucho con ellos, porque siempre están como en otra [onda]...” / C6: “... los pacientes inconscientes, ahí yo no puedo entrar mucho, a pesar de que puedo hablar con los familiares y todo eso, pero con él en sí no puedo...” / C7: “... si ella hubiera estado así perdida [...] no habla, a mí se me hubiera hecho súper difícil..." / C10: "... yo creo que me voy a ir por el adulto, porque con adulto mayor cuesta más la comunicación, hay que repetir muchas veces, ya hay un poco más de demencia senil, entonces no captan bien las ideas y yo creo que eso es lo que más me dificulta..."

Aparecen reiteradamente en el discurso algunas cualidades circunstanciales del sujeto, tales como su estado de ánimo. Observamos una predilección por quienes se muestran alegres y dispuestos, en desmedro de quienes se ven con desgano o desánimo. Nos llama la atención que, siendo el desgano y el desánimo condiciones que comúnmente están presentes en personas cuya salud se encuentra desmedrada, el estudiante se aleje o reprima un acercamiento mayor con ellos.

C1: “... a veces ellos están aburridos de estar mirando para al techo todo el día... y que nadie les diga nada..." / C3: "Es que hay pacientes que son así como... 'ahí viene la señorita' $y$ de inmediato muestran simpatía.” / C7: “... tuve mala experiencia fue un día que me dieron un paciente que tenía que hacerle una visita $y$ estaba súper irritado, enojado.” / C9: “...hay pacientes como enojones, como que no quieren que los toquen." / C6: “... bueno, influye la parte de cómo esté el paciente en eso, de cómo se siente..." / C7: “... lo que me ha dificultado ha sido el estado de ánimo del paciente, de repente despertarlo, de que quería seguir durmiendo, y/o que está de mal humor, de repente está irritable... o los pacientes que no hablan” / C7: “... los pacientes que no hablan, eso igual es súper desagradable porque uno no sabe cómo..." / C7: “...el no quería hablar, no sé, yo le preguntaba ¿está bonito el día?, el me decía 'sí. Si se lo 
preguntaba de nuevo. Me decía 'no'. Entonces no me tomaba en serio." / C7: “... si me encuentro con un paciente que ya no quiere hablar, no sé, he ido dos veces a acercarme donde él y no me quiere hablar, lo dejo no más."

En otro plano, el sujeto hospitalizado puede representar en el aprendizaje un agente solidario activo, facilitando el acceso a antecedentes privados que otorgan al estudiante posibilidades de estructurar una historia clínica completa, y también cimentar su edificación cognitiva en la confianza y la seguridad que brinda una especie de acogida.

C9: “...llegas como un pollito y los pacientes te empiezan a preguntar, señorita qué pasó con este examen, señorita con esto, con lo otro..." / C5: “...Del paciente, yo creo que en general todos tienen como confianza con nosotros los estudiantes, porque donde ven que le podemos dedicar tiempo, se siente como más en confianza con nosotros".

Por otra parte, pareciera no haber preferencias referentes al sexo o la edad, aunque podría existir una mayor inclinación a brindar atención a pacientes del mismo sexo, particularmente en el caso de las alumnas, quienes admiten trabajar con mayor facilidad en tal caso. Las preferencias individuales sobre sexo o edad pude obedecer a asociaciones mentales con personas conocidas, o bien, con situaciones individuales o familiares, favorables o adversas.

C2: “...que sea hombre o mujer me da lo mismo.” / C3: “...me cuesta menos entablar una conversación con una mujer que con un hombre.” / C5: “...prefiero que sea una mujer, por el hecho de que es más fácil que yo sea mujer relacionarme con ella." / C10: “...si puedo escoger entre un adulto y un adulto mayor, yo creo que me voy a ir por el adulto, porque cuesta menos la comunicación..." / C2: “...lo que dificulta mi relación es la edad, los adultos mayores por ejemplo, no así con los niños.” / C2: “...para mí es más cómodo adulto mayor..." / C4: "Los ancianos, los adultos como eso, los niños igual me gustan...” / C7: “...tengo una especial atracción por los adultos mayores, me encantan...”

El elemento 'estado higiénico' aparece de forma explícita como un factor irrelevante, quizás en un intento por persuadirse a sí mismos de emitir un discurso aceptable socialmente. Quizás el cuidar de sujetos deficitarios en higiene desencadene una voluntad de poder, propiciada por sentimientos caritativos como la compasión, operando como un satisfactor de autoafirmación, en la necesidad de verse como alguien últil para el sistema de salud y para otras personas.

C3: "No me importa la hediondez.” / C4: “...a mí no me importa lo de los pañales y menos lo de la pediculosis...” / C9: “... si está hediondo, lo lavo (sonríe)..."

Al parecer, la visita que reciben los pacientes por parte de sus familiares es vista como un obstáculo en la relación que se establece con el paciente, quien prefiere la compañía del visitante, situación que generaría en él sentimientos de frustración. La visita aparece como una interrupción en la relación que se ha formado con el sujeto de cuidado, debiendo postergar el plan de atención que el estudiante había planificado para el día. Esto nos remite a la idea de primacía de las necesidades y expectativas que el estudiante tiene por sobre las del sujeto que se cuida, desde la óptica estudiantil.

El estudiante tiene un 'querer hacer' que considera inhibido por la presencia de los familiares, o por la preferencia natural del sujeto hacia ellos. Aparece la imagen de coalición, en el proceso terapéutico (y de aprendizaje) que es 'interrumpido’ por la visita familiar, al mismo tiempo que el contacto familiar es interrumpido por la presencia extraña de un estudiante. Podría hablarse entonces de la posibilidad de sentir una atracción especial por aquellas personas que no reciben visitas 
de sus familiares. Dicho sujeto cubrirá de mejor manera las necesidades de aprendizaje en el alumno, lo que generaría satisfacción emocional como parte de la construcción de su identidad profesional.

C1: “...querían estar con sus familiares y uno estaba ahí como interrumpiendo y eso dificulta un poco, por lo menos aqui era como a las 11:30, uno no podía hacer nada más porque llegan los familiares y están con el paciente y uno como que tiene que hacer otras cosas y como que se ve cortado un poco, todo lo que uno planifica para su mañana o las cosas que quiere hacer, como que hay que tratar de arreglárselas y de alguna forma tratar de hacerla antes de las 11:30 a.m..." / C9: "...querían estar con sus familiares y uno estaba ahí como interrumpiendo."

\section{Cualidades del estudiante}

El estudiante de Enfermería, como cada persona, posee características en su forma de ser, de pensar y de sentir. Estas cualidades de su personalidad se asocian fuertemente con la relación o vínculo que logra establecer con el paciente, ya que propende o bloquea la comunicación terapéutica y la confianza para acceder a la historia personal. Pueden ser utilizadas como herramientas que faciliten el relacionarse con los demás, convirtiéndose de este modo a sí mismos en instrumentos terapéuticos, que pueden desenvolverse mejor si se valen del potencial que les aporta su personalidad. En este tenor, aparece en el discurso el elemento 'escucha' como factor que orienta la selección de los estudiantes hacia aquellos pacientes que están dispuestos a contar su historia de vida, quedando exceptuados quienes son más introspectivos, silenciosos o indiferentes.

C5: “...yo creo que establezco buena relación con los pacientes, aunque es poquito el tiempo que estamos, crear lazos con ellos, no sé si es por habilidades personales que soy buena para conversar con ellos, hablar...” / C6: “...quizás me ayuda harto la personalidad que tengo y en cuanto a lo sociable que puedo llegar a ser con las personas...” / C7: “... soy muy cariñosa, súper carismática, los pacientes perciben eso...” / C3: “...me gusta escuchar a la gente, me gusta escuchar lo que quieren decir, sus problemas..."

Se observa la timidez como una barrera entre el estudiante y el paciente, transformándose en un obstáculo permanente de la relación y, por ende, del aprendizaje. Esta característica de retraimiento o timidez parece presentarse con mayor claridad durante las primeras experiencias, acompañada de elementos de ansiedad por desempeñarse correctamente y el miedo a equivocarse de manera fatal. Seguramente la ansiedad experimentada durante los primeros días se relaciona con las expectativas que los otros (sujeto de cuidados, personal sanitario, docentes, familiares) han puesto sobre el estudiante, el autoexamen, y el poner a prueba la vocación.

C1: "Mi personalidad a veces dificulta mi relación con los pacientes..." / C3: “...me cuesta establecer relación con los pacientes en algunos casos." / C1: "...pero uno es el que se pone las barreras solo, eso yo encuentro." / C4: “...sí, ha sido medio complicado, porque yo soy como súper sensible...” / C3: “...a mí en general me cuesta entablar relaciones con cualquier persona." / C9: “. . estaba muy nerviosa, entonces era como que había una barrera entre el paciente..."

Seguramente, estas evidencias nos pueden dar luces sobre una preferencia por personas que se muestran abiertas, acogedoras o amables en el proceso de iniciación, centrados en la generosidad y la aceptación de un novato. Ante la falta de experiencia al momento de ingresar a las prácticas clínicas, se observa la tendencia a trabajar con personas que padezcan patologías conocidas por ellos y que al mismo tiempo tengan un bajo grado de 
complejidad.

C14: “...al principio un poco de miedo, porque... es el primer contacto que uno tiene con el paciente, este año, que hace cosas más invasivas que conversar, de repente igual falta un poco de preparación, entonces igual la pone un poco nerviosa..." / C14: “... la primera vez que fui a tomar muestras [de sangre] no me resultó, pero no me puse a llorar ni nada por estilo, no pensé 'no sirvo para esto', pero me han mandado a estudiar para la biblioteca (ríe)." I C10: “...la poca experiencia que tengo, el manejar... técnicas que son simples, pero que igual te ponen nerviosa, entonces eso igual el paciente igual lo percibe." / C8: “... lo que facilita, puede ser que como nos vean jóvenes e inexpertos se abran más con nosotros, porque los pacientes están súper miedosos a decir lo que piensan y a preguntar por lo que tienen, entonces, como nos ven jovencitos nos preguntan a nosotros, como si supiéramos todo, pero en realidad no sabemos casi nada [...] yo encuentro que lo que más facilita es que somos como más cercanos a las personas, el hecho de que no conozcamos tanto, es como que nos ponemos más en el lugar de los pacientes...” / C1: “...escogía pacientes que no estuvieran tan complicados, porque no me sentía seguro a lo mejor de desempeñar una atención buena a ese paciente, a lo mejor no me sentía con los conocimientos necesarios para atenderlo..."

\section{Emociones}

Las emociones se presentan como factores subjetivos e individuales intangibles e imposible de dimensionar, pero aparecen implícitos en el discurso, subsumidos en las categorías precedentes, y también de forma explícita.

De acuerdo a lo relatado por los entrevistados, parecen realizar actos evasivos de las situaciones o sujetos que les producen emociones desagradables, buscando a quienes les produzcan emociones agradables, sentirse satisfechos, útiles, reconfortados, acogidos y motivados por cuadros clínicos emocionantes. Es recurrente en el discurso la relación con personas con cierto grado de dependencia, en tanto genera una situación beneficiosa, tanto para el que cuida como para el que es cuidado, fuente de retribución afectiva mediante el agradecimiento por haber realizado acciones necesarias para el bienestar del sujeto.

Entre las situaciones que se evitan están las que constituyen dificultades clásicas en una asignatura, por miedo a la equivocación, a ser objetados en público o reprobados.

C7: “... lavar a una persona, cambiarle los pañales, que sé yo, todas esas cosas, es como que me pongo bien fría, asi que hay que hacerlo relajado sin tanto dejarme llevar por las emociones que finalmente le juegan en contra." I C5: “... al final siempre me encariño con ellos, y ellos... y si son niños te retribuyen con dibujitos, con regalitos o la abuelita con el pancito que trajo no se quién, siempre se crea una buena relación, tanto de persona como de ayuda al enfermo." C8:"... a mí como que me reconforta harto ayudar a la gente [...] yo me siento súper bien cuando el paciente dice 'gracias señorita', de repente dándole de comer a alguien que no puede comer [...] es súper gratificante y uno se siente bien, como que queda con el corazón llenito. Eso es lo que encuentro más lindo de la carrera." / C10: "Soy como de corazón de abuelita, yo he sentido al menos el primer mes que me vi un poco afectada emocionalmente por lo que le pasada al otro, y de repente como que me costaba desconectarme y me iba para la casa con eso...” / C3: “...al principio tenía miedo, tenía harto miedo, por los comentarios que se daban de otros años, tenía que... el miedo a equivocarse" /C8: "no serviría para trabajar en psiquiatría, los que tienen esquizofrenia, esos me dan como cosa, pero es una cosa mía, porque yo tengo un tío que tiene depresión bipolar y yo casi ni le hablo [...] es tranca mía". / C6: "...del aseo y confort, de esas cosas me preocupo, y que tú veas, en el fondo de que están más necesitados que quizás que otro paciente" / C6: 
"Yo me voy más por la dependencia..." / C1: "...me siento muy conforme con lo que he hecho y también me siento útil[...] como que uno se da cuentan de la labor que hace la carrera y lo que uno está haciendo es un bien para el paciente y es enriquecedor para uno como para el paciente..." / C9: “...el hecho de ayudar como que me da más gratificación.” / C9: “...agradecen, así como muchas gracias, igual nos han dicho, ¡Oh, que son muy amables! y cosas así, entonces es como bien gratificante..." / C9: “... ayudar a las personas, de trabajar con ellas, me entretengo harto en los laboratorios escuchando, cuando de repente los pacientes son felices mientras uno los escucha cuando cuentan todas sus historias.” / C8: “...para mí lo más difícil fue cuando estábamos en segundo, llegar, porque uno no sabe cómo funciona el servicio, uno no sabe a quién dirigirse, uno no sabe nada." / C2: “...encuentro que el ambiente acá es tan malo, como que la gente no está contenta con el trabajo que hace..." / C1: “... la relación que uno mismo tiene con el personal de los servicios porque a veces te dicen cosas o hay mala onda con los estudiantes, a veces eso como que dificulta la relación con los pacientes porque ellos se dan cuenta de todas esas cosas y eso yo creo que puede tener cierta influencia en lo que los pacientes piensen de uno".

\section{DISCUSIÓN Y COMENTARIOS}

La palabra discriminación se puede utilizar para "designar la ruptura de la igualdad que se da en el trato a individuos en un mismo grupo... se pueden distinguir dos tipos de discriminación la 'entre' que denominan positiva y la 'en contra' que denominan negativa" (56); de acuerdo a esto, puede considerarse que los estudiantes de enfermería realizan una discriminación entre sujetos, privilegiando su aprendizaje relacionado con la salud y el cuidado de ellos. El proceso de discriminación que se da en los estudiantes se caracteriza por ser espontáneo, no planifi- cado, ya que ellos no lo identifican. Entonces, este fenómeno opera de forma inconsciente, guiado por atributos intrapersonales y extrapersonales.

En esta aproximación, nos parece que el encuentro con el sujeto que se ha de cuidar se presenta como un momento tan decisorio como breve, que puede ser marcador en el transcurso de las experiencias clínicas y en el desarrollo de un plan de cuidados. Al seleccionar a una persona se confabula una serie de mecanismos, tales como la imagen mental que se tiene de personas conocidas y que se asocia a los rostros visibles al ingresar a una sala de hospitalización. Al racionalizar esta asociación, se obtendría la inferencia de llegar a encontrar acogida en ciertas personas, cuestión que aparece de modo iterativo en los discursos. Entonces, pareciera que el individuo se transforma en símbolo de afecto, de protección, de complicidad, de apertura y un terreno de aprendizaje, más que figurarse en la escena como un ser en sí mismo.

Esta forma de operar respondería a la emocionalidad que se refugia en las propias necesidades de cuidado del novato, y en la expectativa de cursar exitosamente las experiencias clínicas como objeto de evaluación. Pero nos asaltan incisivamente unas interrogantes: ¿aprenden hoy en día las enfermeras a regular su propia emotividad en cuanto a las formas de concentrar o desconcentrar esfuerzos en las personas que cuida?, ¿el cuidado es proveído de acuerdo a las carencias de los individuos o a las propias necesidades afectivas de los profesionales?

Lo que en un primer momento se nos presentaba como un proceso racional, parece esfumarse en la idea de las emociones como motor de unos algoritmos internos no tan racionales. Quizás el componente procesado a la postre por el raciocinio es, primariamente, la sensación, la turbación, la impresión, el desconcierto o la intuición, interrelacionándolos con las posibilidades de subsistir a la prueba de eficacia terapéutica, a la de la mirada atenta de los preceptores, a las de los 
mismos enfermos y al propio juicio sobre la vocación, la capacidad académica y la adaptación a un sistema valórico.

Esta reflexión nos lleva a reforzar nuestras concepciones de aprendizaje vinculadas a un cambio interno. Algo dentro sí se modifica al aprender, lo que no forzosamente se ha de expresar en una conducta. Pero ¿qué se aprende cuando se discrimina por un sujeto que ofrece cualidades propicias para aprender? Creemos que nuestros informantes nos dan luces para comprender este fenómeno como un aprendizaje adaptativo, para llegar a entender desde la familiaridad de lo conocido a la complejidad y extrañeza de lo inexplorado. El aprendiz aprende a habituarse con un ambiente, a familiarizarse con unas rutinas, a incorporar unas creencias, aprende roles, aprende a perder temor y a sentir complacencia al jugar un papel dentro del sistema de cuidados, al pensar y relatar ese papel, y al pensarse en tal papel. En otras palabras, vemos este proceso como parte del forjar una identidad, supuesto en el que fundamos la idea de incentivar los mecanismos para lograr que, en un desarrollo profesional ciertamente íntegro, se deshaga toda forma de parcialidad.

Ciertos factores emergen coligados al mecanismo estudiado, unos vinculados al que cuida, otros al que es cuidado. Características como patología, complejidad, estadía hospitalaria, condiciones mentales y necesidad de cuidado, por nombrar algunos, son factores que crean propensión para el cuidar. Vale decir, el estudiante discrimina de acuerdo a lo que él cree necesario para su aprendizaje, jugando un papel importante en su motivación. Por tanto, el paciente que muestre estas propiedades, por sí solas o en su conjunto, será escogido para ser cuidado. Entonces, una vez más el sujeto de cuidado aparece como un factor central en el cumplimiento de los objetivos que plantea su paso por el campo clínico.

Por otra parte, encontramos que la discriminación se realiza en relación a personas que despiertan emociones agradables, como la motivación, satisfacción. Aparece de forma muy tenue la imagen de personas rechazadas por producir emociones desagradables, lo que refuerza nuestras ideas de discriminación entre individuos y no en contra de ciertos grupos o personas.

De acuerdo a los postulados de Watson (1), nos parece que la habilidad y el gusto por cuidar se halla en los estudiantes en fase de progreso, necesitando primero de una autoafirmación como agente cuidador que le conduzca a una armonía con su rol. Lo que se denomina como 'actitud del cuidado' 6 presenta transformaciones ligadas a las características que señalamos con antelación, encontrando similitudes con las evidencias aportadas por la evidencia, particularmente en lo referido a habilidades comunicativas y al grado de dependencia. No vemos tan nítidamente un correlato con las edades o el nivel educacional, aun cuando fueron elementos que buscamos de forma intencionada en las entrevistas. Ciertamente, algunos de los estudios tomados como referentes $(47,50)$ encuentran reflejo en nuestros hallazgos, los que se refieren a la inclinación por los sujetos comunicativos, los que responden positivamente a un plan terapéutico, a rasgos de personalidad y actitudinales. Aunque el elemento aspecto físico estaba documentado en otros estudios previos $(48,49)$, éste no apareció en los discursos. Quizás la locación elegida para el estudio no ofrecía tipologías de estética especialmente atractivas para los informantes.

Creemos se hace necesario diseñar estrategias de autoconocimiento que haga de éste un proceso consciente, que permita una reflexión sobre el aprendizaje y las formas de aprender, como también de reflexionar so-

\footnotetext{
${ }^{6}$ Valenzuela S. Factores que influyen en la actitud de estudiantes de Enfermería hacia el cuidado de pacientes durante sus experiencias clínicas médico-quirúrgicas.
} 
bre la propia práctica del cuidado en base a la educación emocional.

Los resultados de nuestro estudio, al incluir una recopilación voluminosa de datos y una muestra considerable y heterogénea de informantes, nos hace abandonarnos a la transferibilidad de ellos a poblaciones de similar naturaleza. Sin embargo, más estudios relacionados son necesarios para comprender más ampliamente el fenómeno. El nuestro ha sido limitado a estudiantes de una universidad específica, que comparten muchas características comunes entre sí: la edad, procedencia, nivel educacional, cultural y económico. Será interesante ampliarlo desde la visión de los sujetos hospitalizados, las enfermeras y los profesores de enfermería, complementándolo con otras técnicas sensibles al objeto de estudio: focus groups, observación etnográfica, método biográfico, entre otros.

Agradecimientos: Al Dr. Sergio Toro A. por apoyar tal investigación.

\section{REFERENCIAS}

1. Watson J. Nursing: The Philosophy and Science of Caring. Boston: Little, Brown; 1979.

2. Fawcett J, George J, Walker L, Watson J. Nursing: Human Science and Human Care. A theory of Nursing. Nurs Sci Q. 1985; 2(3): 149-154.

3. Watson J, Smith M. Caring science and the science of unitary human beings: a trans-theoretical discourse for nursing knowledge development. J Adv Nurs. 2002; 37(5): 452-446.

4. Neil R. Teoría del Cuidado de Jean Watson. En: Marriner A, Raile M. Modelos y Teorías en Enfermería. 4a ed. Madrid: Harcourt Brace; 2003.

5. Sanhueza O. El cuidado: Necesidad de Investigar en enfermería. Cienc enferm.
2001; 7(1): 13-22.

6. Carper BA. Fundamental patterns of knowing in nursing. Adv Nurs Sci. 1978; 1(1): 13-23.

7. Van der Zalm JE, Bergum V, Dossetor J. Hermeneutic-phenomenology:providing living knowledge for nursing practice. J Adv Nurs. 2000; 31(1): 211-218.

8. Anderson A. Examining the extent to which Swanson's theory of caring and Carper's ways of knowing can be identified in the process of midwife-led care. RCM Midwives. 2002; 5(10): 340344.

9. Schmidt LA, Nelson D, Godfrey L. A Clinical Ladder Program Based on Carper's Fundamental Patterns of Knowing in Nursing. J Nurs Adm. 2003; 33(3): 146152.

10. Little M. Preparing Nursing Students to be Health Educators: Personal Knowing Through Performance and Feedback Workshops. J Nurs Educ. 2006; 45(3): 131-135.

11. Paley J, Cheyne H, Dalgleish L, Duncan EAS, Niven CA. Nursing's ways of knowing and dual process theories of cognition. J Adv Nurs. 2007; 60(6): 692701.

12. Zander PE. Ways of Knowing in Nursing: The Historical Evolution of a Concept. J Theory Constr Tests. 2007; 11(1): 7-11.

13. Holtslander LF. Ways of knowing hope: Carper's fundamental patterns as a guide for hope research with bereaved palliative caregivers. Nurs Outlook. 2008; 56(1): 25-30.

14. Watson J.Post-hospital nursing: shortage, shifts, and scripts. Nurs Adm Q. 2001; 25(3): 77-82.

15. Arnold R. Pedagogía de la formación de adultos. Montevideo: Cinterfor/OIT; 2004.

16. Vygotski LS. El desarrollo de los procesos psicológicos superiores. $2^{\mathrm{a}}$ ed. Barcelona: Editorial Crítica; 2008.

17. Bermeosolo J. Cómo aprenden los seres 
humanos: Mecanismos psicológicos del aprendizaje. $1^{\text {a }}$ ed. Santiago: Ediciones Universidad Católica; 2005.

18. Ardilla R. Psicología del aprendizaje. $25^{\mathrm{a}}$ ed. México: Siglo XXI editores, S.A.; 2002.

19. González V. Estrategias de enseñanza y aprendizaje. $1^{\text {a }}$ ed. México: Editorial Pax; 2003.

20. Biggs J. Calidad del aprendizaje universitario. $1^{a}$ ed. Madrid: Narcea S.A.; 2004.

21. Pozo JI. Adquisición del conocimiento: Cuando la carne se hace verbo. $1^{\text {a }}$ ed. Madrid: Ediciones Morata S.L; 2003.

22. Cohen J. La inteligencia emocional en el aula. Proyectos, estrategias e ideas. $1^{\text {a }}$ ed. Buenos Aires: Troquel Ediciones; 2003.

23. Ibáñez N. Las emociones en el aula. Estud pedagóg. 2002, 28:31-45

24. Jensen E. Cerebro y aprendizaje. Competencias e implicaciones educativas. $1^{\text {a }} \mathrm{ed}$. Madrid: Narcea S.A; 2004.

25. Antunes CA. Juegos para estimular las inteligencias múltiples. $2^{\text {a }}$ ed. Madrid: Narcea S.A.; 2006.

26. MeyerDK,TurnerJC.Re-conceptualizing emotion and motivation to learn in classroom contexts. Educ Psychol Rev. 2006; 18(1):377-390.

27. Castellà JM, Comelles S, Cros A, Vilà M. Entender(se) en clase: las estrategias comunicativas de los docentes bien valorados. $1^{\text {a }}$ ed. Barcelona: Editorial Graó; 2007.

28. Maturana H. Emociones y lenguajes en educación y política. 9a ed. Santiago: Dolmen ediciones; 2007.

29. Maya A. Conceptos básicos para una pedagogía de la ternura. Bogotá: Editorial Ecoe; 2003.

30. Valenzuela J. Habilidades de Pensamiento y Aprendizaje Profundo; 2007. Hallado en: http://www.uach.cl/rrpp/online/ anexos/2007/06/Habilidades $\% 20$ de $\% 20$ Pensamiento\%20y\%20Aprendizaje\%20 Profundo.pdf [Acceso el 20 diciembre de 2009].
31. Hough MC. Learning, decisions and transformation in critical care nursing practice. Nurs Ethics. 2008; 15(3): 322331.

32. Neville L. Moral difficulties in nursing practice: reflections on the practice of a nurse educator. Nurse Educ Pract. 2004; 4(2): 128-134.

33. Vanlaere L, Gastmans C. Ethics in nursing education: Learning to reflect on care Practices. Nurs Ethics. 2007; 14(6): 758-766.

34. Greenwood D. Relational care: learning to look beyond intentionality to the 'non-intentional' in caring relationship. Nurs Philos. 2007; 8(4): 223-232.

35. Wotton K, Gonda J. Clinician and student evaluation of a collaborative clinical teaching model. Nurse Educ Pract. 2004; 4(2): 120-127.

36. Munhall PL. Epistemology in nursing. En: Munhall PL ed. Nursing Research: A Qualitative Perspective. Sudbury, MA: Jones and Bartlett; 2001.

37. Jackson D, Mannix J. Clinical nurses as teachers: Insights from students in their first semester of study. J C N. 2001; 10(2): 270-277.

38. Papp I, Markkanen M, Von Bonsdorff M. Clinical environment as a learning environment: student nurses' perceptions concerning clinical learning experiences. Nurse Educ Today. 2003; 23(4): 262-268.

39. Scott PA. Emotion, moral perception, and nursing practice. Nurs Philos. 2000; 1(2): 123-133.

40. Bolton SC. Who cares? Offering emotion work as a 'gif' in the nursing labour process. J Adv Nurs. 2000; 32(3): 580586.

41. Bruner JS, Goodnow JJ, Austin A. El proceso mental en el aprendizaje. $1^{\text {a }}$ ed. Madrid: Narcea S.A.; 2001.

42. Barros O. Sistemas de información administrativos. $5^{a}$ ed. Santiago: Editorial Universitaria; 1986. 
43. Sánchez-Pedraza R, Gamboa O, Díaz J. Modelos empleados para la Toma de Decisiones en el Cuidado de la Salud. Rev Salud Pública. 2008, 10(1): 178-188.

44. Rangel M, Escobar C. Teoría y aplicaciones de la toma de decisiones en el sector Salud. Revista de la Facultad de Medicina. Bogotá. 2004; 9(2): 19-24.

45. Corona L, Fonseca M. La toma de decisiones médicas y su formación como habilidad profesional. Rev Cubana Educ Med Sup. 2003; 17(4): 45-52.

46. Mitsunaga BK, Hall BA. Interpersonal attraction and perceived quality of medical-surgical care. West J Nurs Res. 1979; 1(1): 5-20, 24-6.

47. Stockwell F. The unpopular patient. $1^{\mathrm{a}}$ ed. London: Royal College of Nursing; 1972.

48. Dion K. Physical attractiveness and evaluation of children's transgressions. J Pers Soc Psychol. 1972; 24(2): 207-213.

49. Nordholm DLA. Beautiful patients are good patients: Evidence for the physical attractiveness stereotype in first impressions of patients. Soc Sci Med. 1980;
14A, 81-83.

50. Baer ED, Lowery BJ. Patient and situational factors that affect nursing students' like or dislike of caring for patients. Nurs Res. 1987; 36(5): 298-302.

51. Hernández R, Fernández C, Baptista P. Metodología de la Investigación. 4a ed. México: McGraw-Hill; 2004.

52. Taylor S, Bogdan R. Introducción a los métodos cualitativos de investigación. $3^{\mathrm{a}}$ ed. México: Paidós; 2000.

53. Flick U. Introducción a la investigación cualitativa. Madrid: Morata; 2004.

54. Bertaux D. Los relatos de vida en el análisis social. En: Aceves J. Historia Oral. México: Universidad Autónoma Metropolitana; 1993.

55. Strauss A, Corbin J. Grounded Theory in practice. $1^{\text {a }}$ ed. London: Sage Publications; 1997.

56. Fondo de Población de las Naciones Unidas. Resumen del proceso por la no discriminación en Paraguay II; 2005. Hallado en: www.unfpa.org.py/nodiscriminacion/ [acceso el 20 marzo de 2008]. 